\title{
Fluorescence quenching of humic substances by engineering silver nanoparticles
}

Grigoryeva I.O., Konstantinov A.I., Bagdasaryan A., Volkov D.S. Perminova I.V. Lomonosov Moscow State University, Russian Federation, irastarkova@mail.ru

doi: 10.36291/HIT.2019.grigoryeva.115

Silver nanoparticles (Ag-NPs) enter the environment as a result of their intensive use to impart bactericidal properties to the products. In addition, Ag-NPs are used as broadacting antiseptics as an alternative to antibiotics. At the same time, it is of great interest to study the interaction of Ag NPs with the most reactive components of natural ecosystems humic substances. The aim of this work was to evaluate the binding properties of humic substances of coal with respect to silver nanoparticles using the method of quenching of fluorescence.

As HS, we used coal HA (CHP) and their derivatives modified with hydroquinone (CHP-HQ). Quenching experiments used citrated Ag NPs and ionic silver in the form of silver nitrate. Fluorescence spectra were obtained at $\mathrm{pH} 12$ and a carbon concentration of $5 \mathrm{mg} / \mathrm{L}$ to exclude the effect of the internal filter. The silver content ranged from 0 to $5 \times 10^{-5} \mathrm{M}$. The quenching constant was calculated using the Stern-Volmer equation.

It was found that for all HS samples, in the presence of silver ions, fluorescence quenching is observed. The dependence of $\mathrm{F}_{0} / \mathrm{F}$ on $\left[\mathrm{Ag}^{+}\right]$was linear, which allowed the use of the Stern-Volmer equation to calculate the fluorescence quenching constant:

$$
\mathrm{F}_{0} / \mathrm{F}=1+\mathrm{K}[\mathrm{Q}] \text {, }
$$

where $F_{0}$ and $F$ are the fluorescence intensities in the absence and in the presence of a quencher, respectively, [Q] is the quencher concentration.

The quenching constants for HA coal and HA coal modified with hydroquinone were $2.12 * 10^{4} \mathrm{~L} / \mathrm{mol}$ and $2.25 * 10^{4} \mathrm{~L} / \mathrm{mol}$, respectively.

When quenching the fluorescence of Ag-NPs, the dependence of $F_{0} / F$ on [AgNPs] is characteristic of two types of fluorophores, one of which is not available for the quencher [1]. In this case, a modified form of the Stern-Volmer equation was used to calculate the fluorescence quenching constant:

$$
F_{0} / F=1 / f_{1} K[Q]+1 / f_{1}
$$

where $f_{1}$ is the fraction of the initial fluorescence available for quenching.

The quenching constants for $\mathrm{HA}$ coal and HA coal modified with hydroquinone were $3.02 * 10^{6} \mathrm{~L} / \mathrm{mol}$ and $2.10 * 10^{6} \mathrm{~L} / \mathrm{mol}$, respectively. Thus, the values of the quenching constant of the Ag fluorescence of HS nanoparticles exceeded by two orders of magnitude the values of the quenching constant of the fluorescence of HS by ion silver. Nonlinear quenching as a result of adsorption of HV on Ag-NPs.

References

1. Guocheng Zh., Jun Y. // J. Fluorescence. 2017. 27(4):1233-1243. 